\title{
Comparative Analysis of Mesenchymal Stromal Cells Biological Properties
}

\author{
Angela De Luca, ${ }^{1}$ Rosanna Verardi, ${ }^{2}$ Arabella Neva, ${ }^{2}$ Patrizia Benzoni, \\ Elisabetta Crescini, ${ }^{1}$ Er Xia, ${ }^{1}$ Camillo Almici, ${ }^{2}$ Stefano Calza, ${ }^{3}$ and Patrizia Dell'Era ${ }^{1}$ \\ ${ }^{1}$ Fibroblast Reprogramming Unit, Department of Molecular and Translational Medicine, University of Brescia, 25123 Brescia, Italy \\ ${ }^{2}$ Stem Cell Lab-SIMT, A.O. Spedali Civili, Piazzale Spedali Civili 1, 25123 Brescia, Italy \\ ${ }^{3}$ Biostatistics Unit, Department of Molecular and Translational Medicine, University of Brescia, 25123 Brescia, Italy
}

Correspondence should be addressed to Patrizia Dell'Era; dellera@med.unibs.it

Received 25 January 2013; Accepted 28 February 2013

Academic Editors: A. Chapel, I. E. Hoefer, S. M. Hwang, and B. Rogister

Copyright (C) 2013 Angela De Luca et al. This is an open access article distributed under the Creative Commons Attribution License, which permits unrestricted use, distribution, and reproduction in any medium, provided the original work is properly cited.

\begin{abstract}
The stromal progenitors of mesodermal cells, mesenchymal stromal cells (MSCs), are a heterogeneous population of plastic adherent fibroblast-like cells with extensive proliferative capacity and differentiation potential. Human MSCs have now been isolated from various tissues including bone marrow, muscle, skin, and adipose tissue, the latter being one of the most suitable cell sources for cell therapy, because of its easy accessibility, minimal morbidity, and abundance of cells. Bone marrow and subcutaneous or visceral adipose tissue samples were collected, digested with collagenase if needed, and seeded in Iscove's medium containing $5 \%$ human platelet lysate. Nonadherent cells were removed after 2-3 days and the medium was replaced twice a week. Confluent adherent cells were detached, expanded, and analyzed for several biological properties such as morphology, immunophenotype, growth rate, senescence, clonogenicity, differentiation capacity, immunosuppression, and secretion of angiogenic factors. The results show significant differences between lines derived from subcutaneous fat compared to those derived from visceral fat, such as the higher proliferation rate of the first and the strong induction of angiogenesis of the latter. We are convinced that the identification of the peculiarities of MSCs isolated from different tissues will lead to their more accurate use in cell therapy.
\end{abstract}

\section{Introduction}

By definition mesenchyme is an embryonic tissue consisting of loosely packed, unspecialized cells set in a gelatinous ground substance, from which connective tissue, bone, cartilage, and the circulatory and lymphatic systems develop. Indeed, mesenchymal multipotent stromal cells (MSCs) have been shown to reside within the connective tissue of the most organs. In particular, the first and most rich source for isolation of MSCs has been the bone marrow (BM) of different adult organisms. These cells were initially characterized as plastic adherent, fibroblastic-shaped, with a multipotency differentiation capacity both in vitro and in vivo. In 2006 the International Society for Cellular Therapy (ISCT) has suggested a number of phenotypic and functional characteristics to identify mesenchymal stem cells including coexpression of several CD antigens and differentiation into multiple connective tissue cell types such as chondrocytes, adipocytes, and osteocytes [1]. Since then and because of their plasticity, BM-derived MSCs represent an attractive source for cell therapy applications and for studying the mechanism of tissue regeneration. The discovery that the nonadipocyte, stromal fraction of adipose tissues (ATs) contains an abundant population of multipotent progenitors has identified a novel source of cells for therapeutic use. Indeed, both subcutaneous and visceral adipose tissue could be easily collected from different sites during clinical practice in a minimally invasive procedure. AT-derived MSCs are ideal for cellular therapy applications because they can be harvested, multiplied, and handled easily, efficiently, and noninvasively. Moreover morbidity to donors is considerably less, requiring only local anesthesia and exhibiting a short wound healing time [2].

Although the physical contribution of resident MSCs to tissue regeneration is still on debate, it has been clearly shown that implanted MSCs promote tissue repair by secreting 
factors that enhance proliferation of endogenous progenitors, decrease inflammatory response, and favor the angiogenesis process [3]. Nevertheless, BM-, AT-, and umbilical cord blood-derived MSC showed characteristic gene expression signatures [4], thus suggesting that, despite the criteria proposed by ISCT for MSC characterization [1], the various cell lines possess different biological properties.

Then, since MSCs are a sure promise for the future of stem cell-based therapeutic strategies, it would be necessary to identify the characteristics of each cell line in order to use the one that best responds to the specific treatment requirement.

Following this idea, we characterized some biological properties of mesenchymal cells isolated from subcutaneous and visceral adipose tissues. Their comparison with the "gold standard" BM-derived MSCs and with a common fibroblast cell line has evidenced the peculiarities that distinguish and characterize AT-derived MSCs.

\section{Materials and Methods}

2.1. Platelet Lysate Preparation. Platelet lysate pool (PLP) was obtained from the discarded buffy coat fractions (BC) after automatic separation of red blood cells and plasma. Briefly the BC of 15-20 homologous volunteer donors was pooled, diluted in 100-200 mL ABO compatible plasma with heparin $(40 \mathrm{U} / \mathrm{mL})$, and centrifuged $\left(340 \mathrm{~g}, 6 \mathrm{~min}, 22^{\circ} \mathrm{C}\right)$ to obtain the platelet-rich plasma (PRP). The PRP was adjusted, with plasma, at a platelet concentration of $1.2 \pm 0.4 \times 10^{6} / \mu \mathrm{L}$, frozen at $-80^{\circ} \mathrm{C}$ for $24-72 \mathrm{~h}$, and thawed at $37^{\circ} \mathrm{C}$ for $10 \mathrm{~min}$ to lyse the platelets. After a second centrifugation $(4000 \mathrm{~g}$, $15 \mathrm{~min}, 22^{\circ} \mathrm{C}$ ) aimed to remove platelet bodies or debris, the $\mathrm{PLP}$ obtained was aliquoted and stored at $-25-30^{\circ} \mathrm{C}$ [5-7].

2.2. Cell Culture. Bone-marrow-derived MSCs were obtained from bag washouts after scheduled bone marrow harvests according to standards of National Marrow Donor Program. Total nucleated cells from filter and bag residues were obtained after two washings with 100-150 mL PBS and centrifugation at $200 \mathrm{~g}$ for $10 \mathrm{~min}$. The pellet material was gently resuspended in $20-30 \mathrm{~mL}$ PBS and separated by Ficoll-Hypaque gradients centrifugation (Lympholyte-H, Biosera) to yield mononuclear cells. The mononuclear cells were cultured in $25 \mathrm{~cm}^{2}$ culture flasks until they reached confluence and were defined passage 0 . Subsequent passages were seeded at 4000 cells $/ \mathrm{cm}^{2}$.

Cells were grown in Iscove's medium (Sigma) containing Penicillin $(100 \mathrm{U} / \mathrm{mL}) /$ Streptomycin $(100 \mu \mathrm{g} / \mathrm{mL})(\mathrm{P} / \mathrm{S})$, $200 \mathrm{mM}$ L-glutamine, $25 \mu \mathrm{g} / \mathrm{mL}$ fungizone, and 5\% PLP (MSC medium).

Adipose-derived MSCs were isolated from visceral (OMSC, omental) or subcutaneous (L-MSC, lipoaspirate) adipose tissues of different donors (middle age). Omental samples (25-30 g) were transferred in sterile PBS, repeatedly washed to remove blood residuals, minced with scissors and scalpels, centrifuged to remove liquid phase, and digested with $0,075 \%$ collagenase IA (Sigma) at $37^{\circ} \mathrm{C}$ for $40-50 \mathrm{~min}$. Enzyme activity was neutralized by addition of Iscove's medium (Sigma) containing 20\% fetal bovine serum (FBS,
Biosera). Lipoaspirates were diluted in PBS and the fat phase digested as above. Dispersed cells were centrifuged $10 \mathrm{~min}$ at $1200 \mathrm{~g}$, resuspended in Iscove's medium containing $\mathrm{P} / \mathrm{S}, 200 \mathrm{mM}$ L-glutamine, $25 \mu \mathrm{g} / \mathrm{mL}$ fungizone, 5\% PLP, filtered $(100 \mu \mathrm{m})$ to remove undigested tissue, and plated at 50,000 cells $/ \mathrm{cm}^{2}$ in $75 \mathrm{~cm}^{2}$ culture flasks. Subcultures were seeded at 2,000 cells $/ \mathrm{cm}^{2}$ [8].

Human foreskin fibroblasts (HFFs) were provided by ATCC (Number: CRL-2429) and were grown in DMEM (Sigma), 10\% FBS.

2.2.1. Growth Kinetics. For the assessment of growth kinetics MSCs at P4 and HFF were seeded at $2 \times 10^{3}$ cells $/ \mathrm{cm}^{2}$ in 24 -well plate. Every day, over 2 weeks, cells from three pooled wells were trypsinized, resuspended in PBS containing 5\% FBS in TRUCOUNT tubes (BD Biosciences), and analyzed with flow cytometer (FACSCanto Diva, BD Bioscience). Viability was assessed by addition of 7-aminoactinomycin D (7-AAD, BD Biosciences). Population doubling time (h) was calculated using the algorithm provided by http://www.doubling-time.com/.

2.2.2. Senescence Assay. Cells were seeded at $5 \times 10^{4}$ cells $/ \mathrm{cm}^{2}$ in 24-well plate and incubated in standard conditions. After 2 days, cells were fixed with $1 \%$ glutaraldehyde solution for 5 min and the $\beta$-galactosidase $(\beta$-gal) enzymatic activity at $\mathrm{pH} 6.0$, that excludes the lysosomal isoform, was visualized by staining cells with the chromogenic substrate 5-bromo-4chloro-3 indolyl- $\beta$-d-galactopyranoside (X-gal). The number of $\beta$-gal positive cells in three random microscopic fields was evaluated.

2.2.3. Immunophenotypic Analysis. BM-MSC, L-MSC, OMSC, and HFF were trypsinized and resuspended in flow cytometry buffer (PBS pH 7.2, FBS $2 \%$, sodium azide $0.01 \%$ ). Single cell suspensions $\left(5 \times 10^{5}\right.$ cells $\left./ 100 \mu \mathrm{L}\right)$ were incubated $20 \mathrm{~min}$ with the conjugated monoclonal or isotype-matched IgG control antibodies. After extensive washes, cells were analyzed by flow cytometry. Cellular viability was assessed by incubating cell suspension with plasma membrane permeability marker 7-amino-actinomycin D (7-AAD, BD Bioscience). CD45 Pe-Cy7, CD73 PE, CD90 FITC, CD105 PerCPCy5.5, CD31 PE, and CD34 APC, were from BD Biosciences. CD146 PerCP-Cy5.5 and CD105 FITC were from Biolegend, CD133 APC was from Miltenyi Biotec, and anti SSEA4-PE was from R\&D.

2.3. Cell Differentiation. Cell differentiation was achieved using StemPro Adipogenesis, Osteogenesis, and Chondrogenesis differentiation kits (Life technologies), following the manufacturer's instruction. At the end of the differentiation period, all cultures were fixed with $4 \%$ formaldehyde for $30 \mathrm{~min}$ and adipocytes stained with $0,3 \%$ Oil Red O solution for $10 \mathrm{~min}$; bone cells with $2 \%$ Alizarin Red S solution ( $\mathrm{pH}$ 4.2) for $3 \mathrm{~min}$, while micromass cultures of chondrocytes were stained with $1 \%$ Alcian blue solution in $0.1 \mathrm{~N} \mathrm{HCl}$ for 30 minutes. 
TABLE 1: Flow cytometry characterization of MSC populations.

\begin{tabular}{lcccccccccccc}
\hline $\begin{array}{l}\text { Cell } \\
\text { population }\end{array}$ & $\begin{array}{c}\text { Passage } \\
\text { number }\end{array}$ & $\begin{array}{c}\text { CD45 } \\
(\%)\end{array}$ & $\begin{array}{c}\text { CD105 } \\
(\%)\end{array}$ & $\begin{array}{c}\text { CD90 } \\
(\%)\end{array}$ & $\begin{array}{c}\text { CD73 } \\
(\%)\end{array}$ & $\begin{array}{c}\text { CD146 } \\
(\%)\end{array}$ & $\begin{array}{c}\text { CD133 } \\
(\%)\end{array}$ & $\begin{array}{c}\text { CD34 } \\
(\%)\end{array}$ & $\begin{array}{c}\text { CD31 } \\
(\%)\end{array}$ & $\begin{array}{c}\text { MSC } \\
(\%)\end{array}$ & $\begin{array}{c}\text { EPC } \\
(\%)\end{array}$ & $\begin{array}{c}\text { EC } \\
(\%)\end{array}$ \\
\hline L-MSC & 0 & 1.5 & 96 & 97 & 98 & 91 & 0.5 & 1.7 & 2.4 & 95 & 0.3 & 2.5 \\
& 3 & 0.1 & 95 & 98 & 97 & 93 & 0.3 & 0.3 & 0.3 & 95 & $<0.1$ & 0.7 \\
O-MSC & 0 & 18 & 74 & 86 & 90 & 72 & 0.4 & 2.3 & 2.8 & 64 & $<0.1$ & $<0.1$ \\
& 3 & 0 & 83 & 92 & 89 & 98 & 0.1 & 3.1 & 0.9 & 80 & $<0.1$ & $<0.1$ \\
BM-MSC & 0 & 8 & 93 & 95 & 96 & 94 & 1.1 & 3.5 & 0.5 & 94 & 0 & $<0.1$ \\
& 3 & 4 & 98 & 98 & 99 & 93 & 0.8 & 0.2 & 0.3 & 98 & 0 & 0 \\
HFF & u.d. & 0 & 100 & 100 & 100 & 31 & 0 & n.d. & 0 & 100 & 0 & 0 \\
\hline
\end{tabular}

MSCs were defined by coexpression of CD105, CD90, and CD73; EPC were defined by coexpression of CD133, CD146, CD105, and CD31; and EC were defined by coexpression of $\mathrm{CD} 34, \mathrm{CD} 146, \mathrm{CD} 105$, and $\mathrm{CD} 31$. The reported values represent intensity mean of two independent experiments. u.d.: undefined; n.d.: not determined.

2.4. RNA Extraction, Semiquantitative and Quantitative RTPCR Analysis. Total RNA was extracted from MSC as described [9]. Contaminating DNA was digested using DNAse, following indications reported in RNeasy Micro Handbook (Qiagen). Two $\mu \mathrm{g}$ of total RNA were retrotranscribed with MMLV reverse transcriptase (InVitrogen) using random hexaprimers in a final $20 \mu \mathrm{L}$ volume. Quantitative PCR (qPCR) was performed with a Biorad iCycler iQ Real-Time PCR Detection System using a iQ SYBR Green Supermix (Biorad) according to the manufacturer's instructions. The qPCR specific primers (final concentration $400 \mathrm{nM}$ ) were as follows: GAPDH for: $5^{\prime}$-GAAGGTCGGAGTCAACGGATT; rev: $5^{\prime}$-TGACGGTGCCATGGAATTTG; Oct4 for: $5^{\prime}$-GGGTTTTTGGGATTAAGTTCTTCA; rev: 5’-GCCCCCACCCTTTGTGTT; Nanog for: 5'-AGGAAGACAAGGTCCCGGTCAA; rev: $5^{\prime}$-TCTGGAACCAGGTCTTCACCTGT; Sox2 for: $5^{\prime}$-CAAAAATGGCCATGCAGGTT; rev: $5^{\prime}$-AGTTGGGATCGAACAAAAGCTATT; Klf4 for: 5' -AGCCTAAATGATGGTGCTTGGT; rev: 5'-TTGAAAACTTTGGCTTCCTTGTT. Gene expression levels were evaluated using BM-MSC as reference cell line. Data were analyzed using REST [10].

2.5. Immunosuppression Assay. Peripheral blood mononuclear cells (PBMCs) were isolated from the blood of healthy donors by Ficoll gradient, resuspended in RPMI 1640 (Sigma) supplemented with $10 \%$ FBS, Penicillin (100 U/mL)/Streptomycin $(100 \mu \mathrm{g} / \mathrm{mL})(\mathrm{P} / \mathrm{S}), 200 \mathrm{mM} \mathrm{L}$-glutamine, $25 \mu \mathrm{g} / \mathrm{mL}$ fungizone, and labeled with the vital dye carboxyfluorescein diacetate, succinimidyl ester (CFSE, 0,8 $\mu \mathrm{M}$, Sigma) for $20 \mathrm{~min}$ at $37^{\circ} \mathrm{C}$. Then, $2 \times 10^{5} \mathrm{CFSE}$-loaded cells were seeded on irradiated ( $30 \mathrm{~Gy}$ ) MSC or HFF plated at $2.5 \times$ $10^{4}$ cell/well in U bottom 96-well plate. PBMC proliferation was stimulated by addition of $5 \mu \mathrm{g} / \mathrm{mL}$ phytohemagglutinin (Sigma) for 5 days. The serial halving of the fluorescence intensity of CFSE, that corresponds to cell division, was evaluated by flow cytometry [11]. Data were analyzed with FlowJo program.

2.6. Endothelial Cell Sprouting Assay. Human umbilical vein endothelial cells (HUVECs) have been aggregated as spheroid bodies in M199 (Sigma) culture medium containing $10 \%$ FBS and 20\% methylcellulose (Sigma), in a round bottom 96-well plate, 1000 cells/well. Under these conditions all suspended cells contribute to the formation of a single endothelial cell spheroid. After $18-24 \mathrm{~h}$ the HUVEC spheroids (20 spheroids/sample) were embedded into collagen gel (Roche) and incubated at $37^{\circ} \mathrm{C}$ to polymerize for $1 \mathrm{~h}$. The layer of collagen gel/spheroids has been covered with culture medium containing conditioned medium (CM) from the various cell lines. The number of sprouts per spheroid was analyzed using ImageJ software (http://rsb.info.nih.gov/ij/) and compared with those induced by VEGF $(30 \mathrm{ng} / \mathrm{mL})$. At least two replicates were included within each experiment.

$\mathrm{CM}$ was obtained by seeding cells at $2.6 \times 10^{4}$ cells $/ \mathrm{cm}^{2}$ and incubating cultures for $48 \mathrm{~h}$ in standard conditions. CMs were collected, clarified by centrifugation, concentrated using $3000 \mathrm{~K}$ Centricon (Millipore), and stored at $-80^{\circ} \mathrm{C}$ until used.

\section{Results}

3.1. MSC Isolation and Characterization. Recently, adipose tissue (AT) has been proposed as a source of therapeutic stromal cells with functional characteristics overlapped to that of BM-derived MSC; indeed, the large number of cells isolated from AT and their higher proliferation rate early responds to the request of cells to be used for therapeutic purposes. To address some of the open questions concerning the biological properties of these cells, we isolated adiposederived mesenchymal stromal cells from surgical lipoaspirates (L-MSC) or from omentum (O-MSC) by collagenase digestion of the tissue, while BM-MSCs were obtained from bag washouts after scheduled bone marrow harvest. MSCs within the samples were allowed to adhere to a cell culture dish and maintained in culture for several passages, using a PLP-containing medium. Once cell lines were established, the different MSC populations were characterized following the criteria suggested by the International Society for Cellular Therapy [1]. Flow cytometry was used to assess cell viability (7AAD exclusion) and to evaluate the percentage of cultured cells that do not belong to the hematopoietic lineage $\left(\mathrm{CD} 45^{-}\right)$, but coexpress MSC antigens CD105, CD90, and CD73 (Table 1). Since endothelial cells share 
TABLE 2: Proliferation properties of MSC.

\begin{tabular}{|c|c|c|c|c|c|}
\hline Cell Population & Doubling time $(\mathrm{h})$ & Density at confluence & Cell senescence & Clones (limiting dilution) & Clones (CFU) \\
\hline L-MSC & 18.76 & 140,000 & $15.2 \%$ & $1.44 \%$ & $9 \%$ \\
\hline O-MSC & 34.03 & 44,000 & $11.45 \%$ & $0.66 \%$ & $0 \%$ \\
\hline BM-MSC & 35.07 & 50,000 & $30.00 \%$ & $0.22 \%$ & $12 \%$ \\
\hline $\mathrm{HFF}$ & 17.06 & $>200,000$ & $4.63 \%$ & $0.00 \%$ & $>50 \%$ \\
\hline
\end{tabular}

The reported values represent the mean of two independent experiments.

some of the MSC markers, the presence of both precursor $\left(\mathrm{CD} 133^{+} / \mathrm{CD}_{105^{+}} / \mathrm{CD} 146^{+} / \mathrm{CD} 31^{+} / \mathrm{CD} 45^{-}\right)$and mature $\left(\mathrm{CD} 34^{+} / \mathrm{CD} 105^{+} / \mathrm{CD} 46^{+} / \mathrm{CD} 31^{+} / \mathrm{CD} 45^{-}\right)$endothelial cells was evaluated in parallel. The same analysis was repeated later on and, although the percentage of starting MSC population was high, it further increases after the first two passages in culture, ranging from $80 \%$ for O-MSC, up to $97 \%$ for BMMSC (Table 1). Since endothelial cells, both precursors and mature cells, represent, at higher passage, less than $1 \%$ of the total population, our data point to the uniformity of the MSC populations that has been maintained up to P8 (data not shown). Then, all the following experiments were carried out using MSC at P4.

3.2. Proliferative Properties of MSC. The possibility to use MSC as therapeutic agent leads us to culture the cells in a medium free of animal serum. Indeed, by growing cells in $5 \%$ hPLP rather than fetal bovine serum [6], we were able to expand L-MSC at the fifth passage 15322 times (corresponding to approximately 14 duplications; range 1400-76199) in respect to the 422 times (corresponding to approximately 9 duplications; range 16-827) of FBS. The proliferation rates of MSC grown in Iscove's medium containing 5\% hPLP are shown in Figure 1. Doubling time (DT) calculation of their logarithmic growth phase (h) indicates that L-MSC grow faster than O- or BM-MSC (Table 1) and indeed similar results were shown by other groups [12].

Morphologically all the MSCs have a spindle-shaped appearance but L-MSCs are more closely related to HFF, whereas O-MSCs mostly resemble BM-MSC (data not shown). Because of this morphology, cell number at confluence is very different, being at least doubled for L-MSC in respect to O- and BM-MSC (Table 2). Since the lower proliferation rate of $\mathrm{O}$ - and $\mathrm{BM}-\mathrm{MSC}$ could be explained by an increased cellular aging, we analyzed replicative senescenceassociated cytoplasmic $\beta$-galactosidase activity. MSC cultures were fixed, stained with X-gal, and the number of cells containing the cyan dye was evaluated. No correlation was found with cellular senescence and the age of donor. As shown in Table 1, BM-MSC showed the higher percentage of senescent cells, doubling the values found in adipose-derived MSC. Nevertheless, no significant differences were found in L- and O-MSC, thus excluding the hypothesis of an increased senescence for the latter.

3.3. Clonal Properties of MSC. By definition stem cells undergo "unlimited" division because of their self-renewal capacity. To define the presence of stem cells in our populations, we analyzed MSC in colony formation and limiting

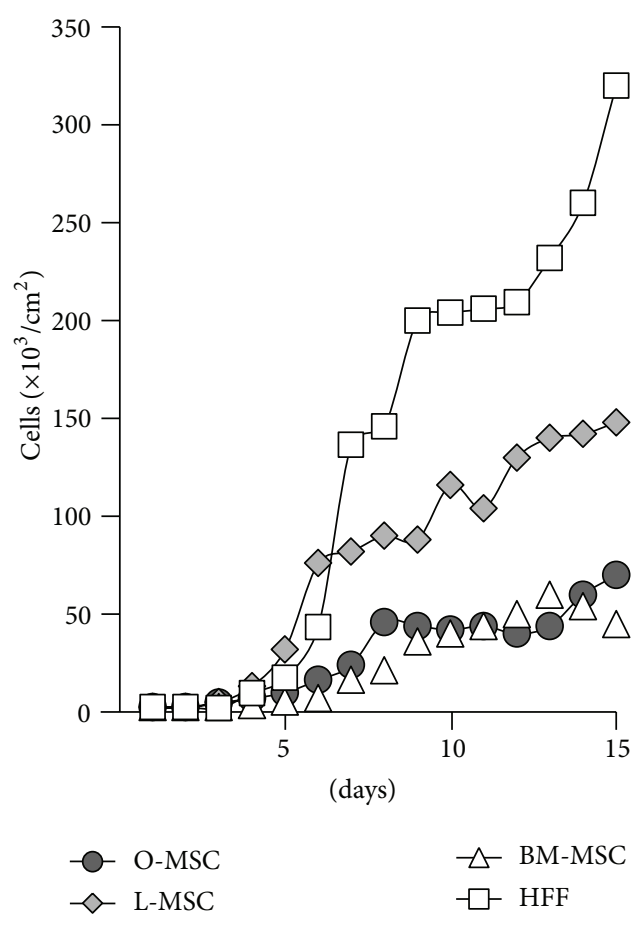

FIGURE 1: Proliferative properties of adipose-derived MSC. Growth curve of MSC populations at P4 in MSC medium. Cells were seeded at $2 \times 10^{3}$ cells $/ \mathrm{cm}^{2}$ and counted daily in triplicate in TRUCOUNT tubes (BD Biosciences), using FACSCanto Diva flow cytometer (BD Biosciences). Data represent the mean of at least two independent experiments.

dilution assays. Both tests evaluate the clonal capacity of a cell but, while in the colony formation assay the cells (100 cells $/ 10 \mathrm{~cm}$ dish) are maintained in a common medium, in the limiting dilution assay every cell (500 cells in 96-well plates) must condition its own environment. The results we obtained are shown in Table 2. In general, the percentage of clonal cells in MSC populations was low, in agreement with literature data [13]. In detail, the number of clones was similar in L- and BM-MSC, while was bending to zero for O-MSC. It should be pointed out that in the limiting dilution assay we obtained a lower number of clones, due to the difficulty of a single cell to survive in an unconditioned environment. Indeed, HFF cells, which are devoid of any stemness competence, do not survive in this condition.

3.4. Differentiation Capacity of MSC. Stemness is also associated with the capacity of the cells to differentiate into 

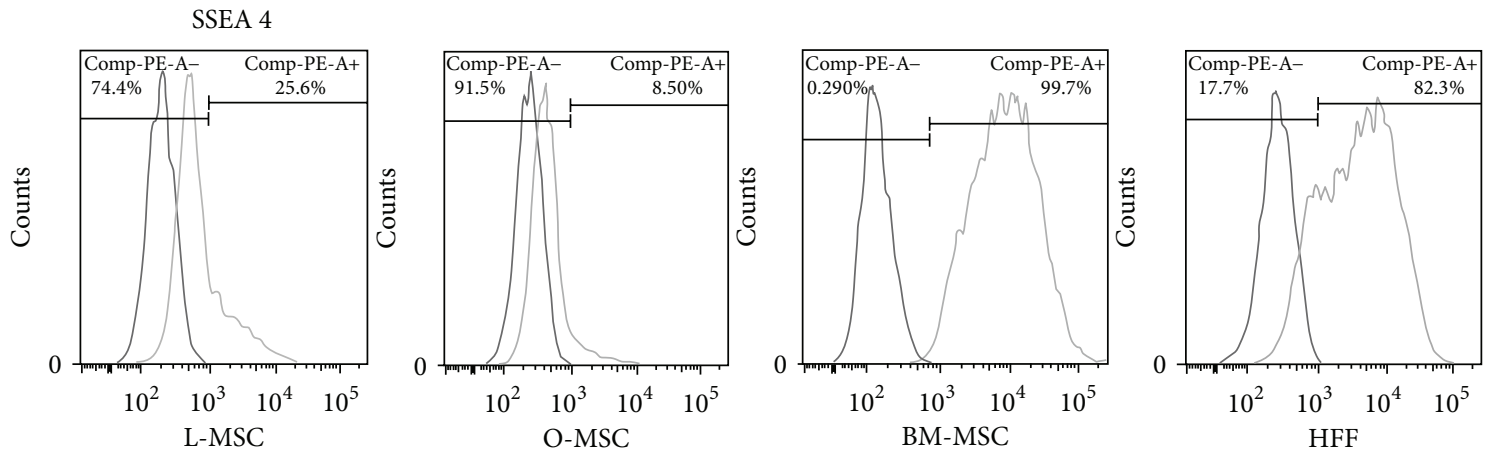

(a)

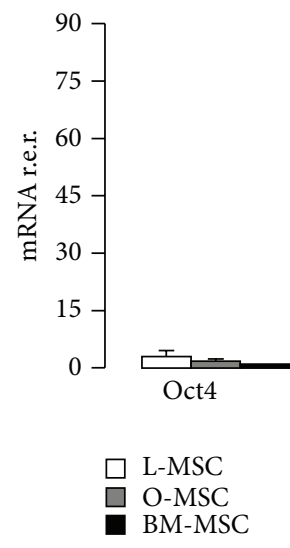

(b)

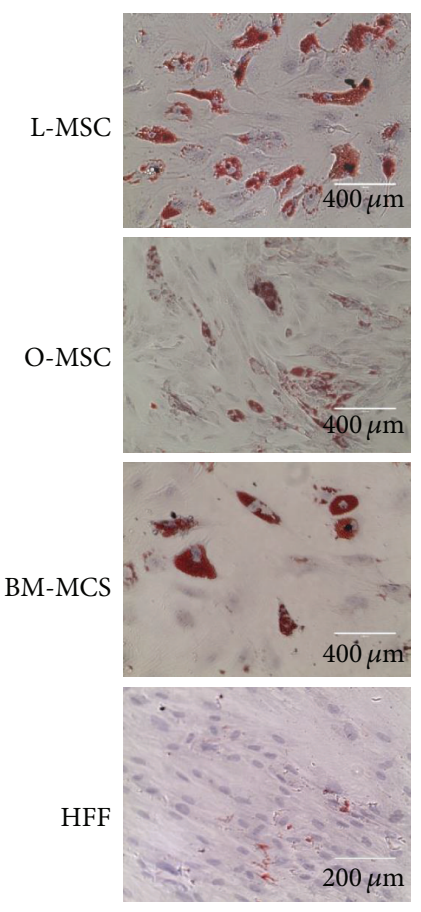

Adipocytes
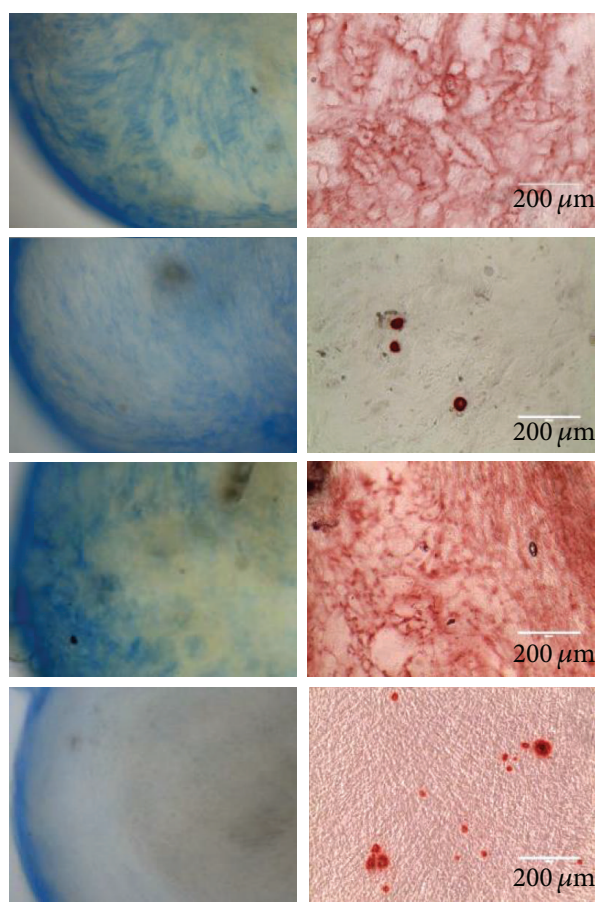

Chondrocytes
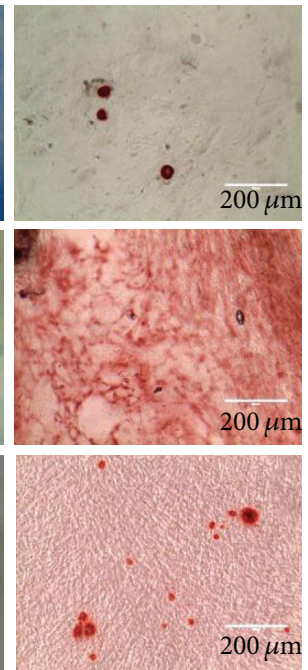

Osteocytes

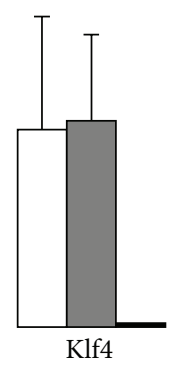

(c)

FIGURE 2: Differentiation capacity of adipose-derived MSC. (a) Cells were detached with trypsin and SSEA4 membrane protein was evaluated by flow cytometry using a PE-conjugated antibody. (b) qPCR analysis of the expression of pluripotency markers in MSC. Total RNA was extracted from cell lines, equivalent amounts of cDNA were amplified by qPCR and gene expression levels were quantified using REST [10]. BM-MSC was used as a reference cell line. Data are representative of at least two independent experiments. (c) Oil Red O staining of MSC after adipogenic induction, Alcian Blue staining after chondrogenic induction, and Alizarin Red staining after osteogenic induction. 


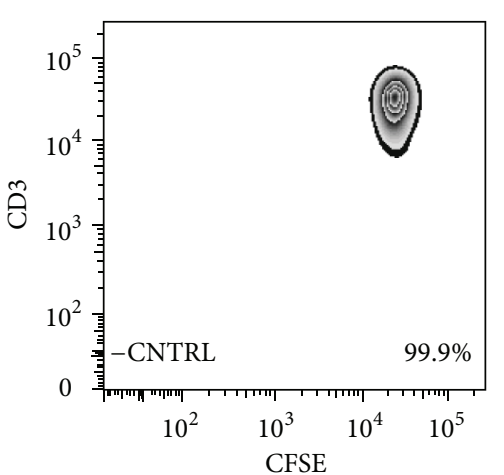

(a)

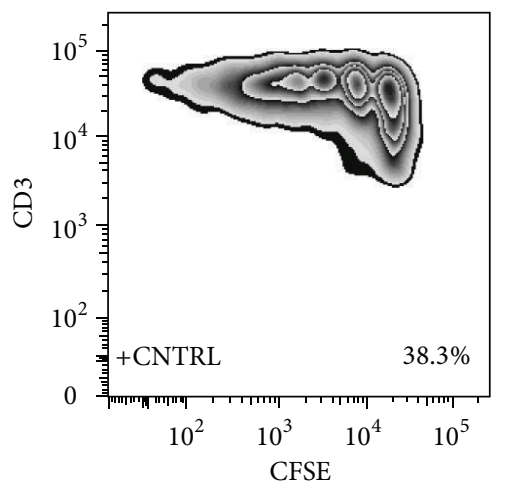

(d)

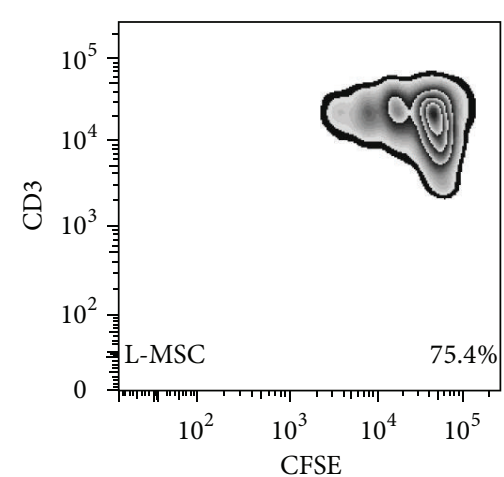

(b)

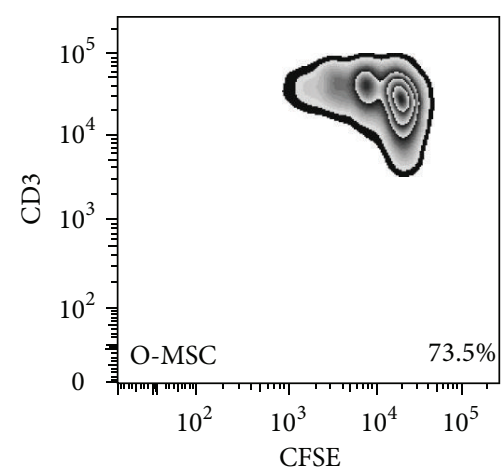

(e)

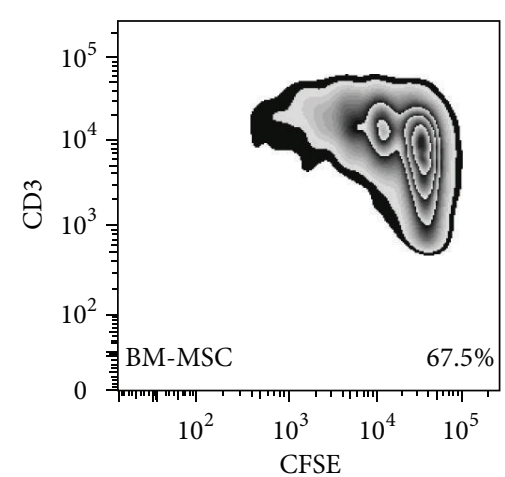

(c)

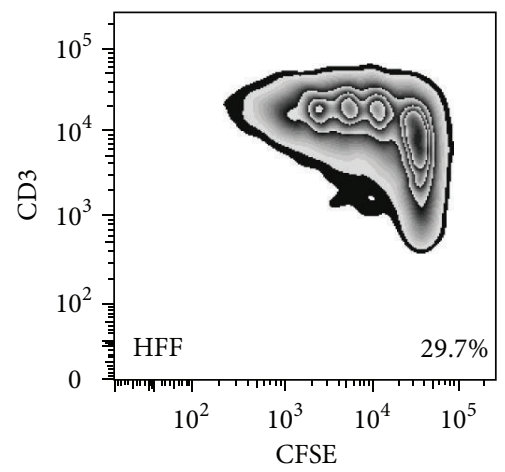

(f)

FIGURE 3: Immunosuppressive capacity of adipose-derived MSC. PBMCs were loaded with CFSE (-CNTRL) and stimulated with PHA (+CNTRL) to induce cell proliferation. CD3 positive cells were evaluated by flow cytometry. Data represent the dilution of CSFE corresponding to cell division. Percentages represent the amount of resting cells.

several lineages and MSCs are often referred as multipotent cells, with a differentiation potential restricted to mesodermal tissues. However, their aptitude, under defined conditions, to transdifferentiate in cells of ectodermal and endodermal origin has been also demonstrated $[14,15]$. In order to investigate the differentiation potential of AT-derived MSC, some of the genes associated with pluripotency have been evaluated by qPCR and flow cytometry. The results illustrated in Figure 2(b) show low levels of Oct4 and Nanog in all MSCs, thus suggesting the absence of pluripotency for these cell lines. Besides, Sox2 and, mostly, Klf4, a transcriptional regulator of adipogenesis [16], are selectively upregulated in AT-derived MSC.

On the other hand, the membrane marker SSEA4 is characteristic of the entire BM-derived population, while is expressed by $25 \%$ of L-MSC population and only by $8.5 \%$ of O-MSC (Figure 2(a)).

Following these results, we tested the canonical differentiation properties of MSC along the adipogenic, chondrogenic, and osteogenic lineages, using StemPro differentiation kits (Life Technologies), together with HFF. The results (Figure 2(c)) show that all the three cell lines easily differentiate in adipocytes and chondrocytes as illustrated by Oil Red O and Alcian Blue staining, respectively. On the contrary, osteogenesis, represented by Alizarin Red staining of calcium deposition, was poorly effective in O-MSC, even with different media (data not shown). A weaker differentiation capacity of the O-MSC in respect to L-MSC is also reported by Toyoda et al. [17]. Because of these results, and following ISCT guidelines [1], we cannot classify O-MSC as mesenchymal stem cells.

3.5. Immunosuppression. The most important property of mesenchymal stem cells, that allows their therapeutic allogenic use in graft versus host disease, is their capacity to suppress activation of immune cells $[18,19]$. Then, PHAstimulated PBMCs were challenged with MSC populations and the cell division of $\mathrm{CD}^{+}$cells was monitored by flow cytometry. The results are shown in Figure 3, where the percentage represents the number of nondividing resting cells. Unstimulated cells represent the negative control, while the positive control is signified by PBMC stimulated with PHA alone. At variance with fibroblasts, all MSC populations, including O-MSC, immunosuppress PHA-induced proliferation of T cells.

3.6. Secretion of Angiogenic Growth Factors. Several papers report that the benefits derived from MSC injection might result from the secretion of several factors including those that stimulate neovascularization of injured tissue [20]. To assess the angiogenic potential of MSC, we investigated the capacity of their conditioned medium (CM) to induce the 

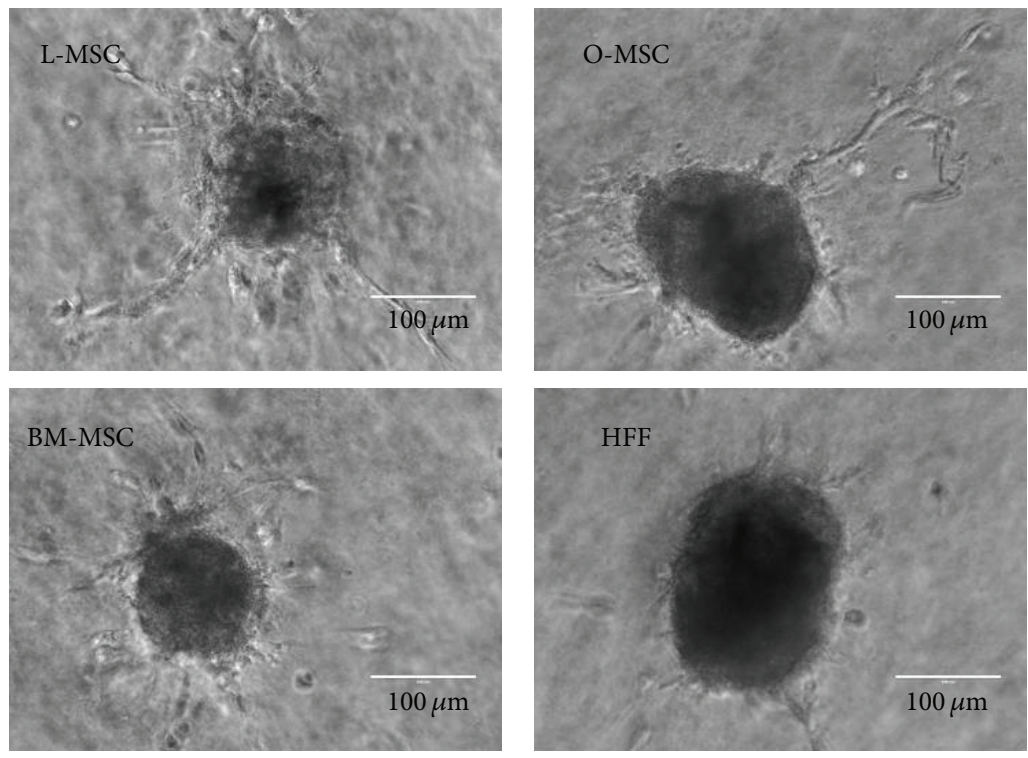

(a)

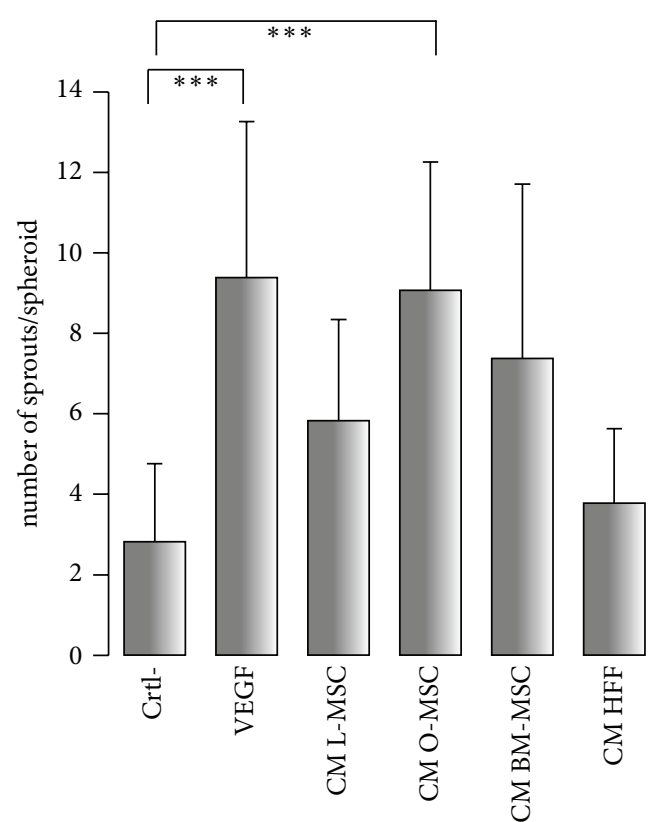

(b)

FIGURE 4: In vitro angiogenesis of MSC conditioned medium. 3D collagen-embedded HUVEC spheroids were stimulated with CM from the reported cell populations. (a) Microscopic observation at $24 \mathrm{~h}$. (b) Representation of modeled counts using a statistic general linear model with exponential weights. ${ }^{* * *}$ Significant differences $(P<0.001)$.

sprouting of collagen-embedded HUVEC spheroids. Results are shown in Figure 4. In agreement with literature data, CM derived from all the MSC populations shows an angiogenic capacity that in the case of O-MSC can be compared to those exerted by the proangiogenic protein vascular endothelial growth factor (VEGF).

\section{Discussion}

The adipose tissue is made of a large number of adipocytes and other nonfat cells, surrounded by connective tissue matrix. The nonadipocyte cellular components include inflammatory cells, immune cells, preadipocytes, and fibroblasts, together with endothelial cells and pericytes which constitute the capillary network [21]. Then, one or more of these cell types must be the source of cultured mesenchymal stem/stromal cells. From recent literature, it has been suggested that blood vessels harbor perivascular native ancestors of the cultured MSC and, depending on the anatomy of the vessel, can be classified as pericyte- or adventitial-derived MSC [22]. Although the International Society for Cellular Therapy (ISCT) has suggested a number of phenotypic and functional characteristics to identify mesenchymal stem cells, an updated classification, following recent literature, is urgently needed. Indeed, the cluster of differentiation (CD) markers we analyzed (CD105, CD90, CD73) are coexpressed by all the mesenchymal-derived cells, including endothelial cells, fibroblasts, and pericytes, thus making this evaluation redundant. In this regard, our data confirm the specificity of CD31, Pecam-1, as a true endothelial cell marker.

In our study, we have considered adipose tissue samples derived from subcutaneous and visceral fat, obtained respectively by lipoaspiration and from omentum. Although other groups have compared AT-MSC from different sources, parallel controls with BM-MSC or fibroblasts were missing. Because of the lack of cell identification markers, it is of outmost importance to refer the results to what is considered a "gold-standard" (BM-MSC) and to extensively describe the biological characteristics of the selected stromal cells.

The application of ISCT guidelines to our cells implies that lonely L-MSC can be ranked as mesenchymal stem cells, because O-MSC, even though we tested different media, lack the capacity to differentiate toward the osteogenic lineage. A weaker differentiation capacity of the omental-derived MSC is also reported by Toyoda et al. [17], although in both cases the samples do not belong to the same patient. Nevertheless, these omental cells, that share all the other properties with the so-called stem cells, hold the highest angiogenic potential, as also shown by other groups [23,24]. Then, from a therapeutic point of view, these cells could be useful in pathologies where cellular differentiation is not required but both immunosuppression and a proangiogenic environment are needed. From the various studies, a number of subpopulations of mesenchymal cells are emerging for which it is absolutely necessary to identify unique markers suitable for their isolation and characterization. In this regard, as reported for mice [25], we showed that also in humans SSEA4 expression is elevated in BM-MSC, but, unfortunately, is expressed also by fibroblasts, thus limiting its effectiveness as a specific cellular marker.

In conclusion, our data showed that cells $\mathrm{CD} 105^{+} / \mathrm{CD}^{+} 0^{+} /$ $\mathrm{CD}^{+} 3^{+} / \mathrm{CD} 45^{-}$isolated from subcutaneous or from omental adipose tissues possess distinct characteristics. In particular, L-MSCs hold the biological and therapeutical properties of 
BM-MSC, such as the multipotent differentiation capacity and the immunosuppressive capability, but, in addition, have a higher proliferation rate. On the other hand, the omental cells, even if devoid of osteogenic differentiation, are also able to suppress $\mathrm{T}$ cell proliferation and, even better, possess the highest proangiogenic capacity. These properties propose L-MSC as a valuable therapeutic substitute of BM-MSC and identify O-MSC as the cells responsible for the widely recognized tissue healing properties of the omentum.

\section{Conflict of Interests}

The authors declare no conflict of interests with respect to the present paper.

\section{Acknowledgments}

The authors are indebted to Riccardo Nascimbeni, Gianluigi Bisleri, and Edoardo Cervi for providing them biological samples. They thank Marco Presta, Livio Dei Cas, Marco Metra, and Stefania Mitola, for helpful discussion. This work was supported by Grants from Fondazione Berlucchi, Ministero Istruzione Università e Ricerca, and Centro per lo Studio del Trattamento dello Scompenso Cardiaco (University of Brescia).

\section{References}

[1] M. Dominici, K. Le Blanc, I. Mueller et al., "Minimal criteria for defining multipotent mesenchymal stromal cells. The International Society for Cellular Therapy position statement," Cytotherapy, vol. 8, no. 4, pp. 315-317, 2006.

[2] R. Ogawa, "The importance of adipose-derived stem cells and vascularized tissue regeneration in the field of tissue transplantation," Current Stem Cell Research \& Therapy, vol. 1, no. 1, pp. 13-20, 2006.

[3] D. J. Prockop, "Repair of tissues by adult stem/progenitor cells (MSCs): controversies, myths, and changing paradigms," Molecular Therapy, vol. 17, no. 6, pp. 939-946, 2009.

[4] W. Wagner, F. Wein, A. Seckinger et al., "Comparative characteristics of mesenchymal stem cells from human bone marrow, adipose tissue, and umbilical cord blood," Experimental Hematology, vol. 33, no. 11, pp. 1402-1416, 2005.

[5] K. Schallmoser, C. Bartmann, E. Rohde et al., "Human platelet lysate can replace fetal bovine serum for clinical-scale expansion of functional mesenchymal stromal cells," Transfusion, vol. 47, no. 8, pp. 1436-1446, 2007.

[6] C. Capelli, M. Domenghini, G. Borleri et al., "Human platelet lysate allows expansion and clinical grade production of mesenchymal stromal cells from small samples of bone marrow aspirates or marrow filter washouts," Bone Marrow Transplantation, vol. 40, no. 8, pp. 785-791, 2007.

[7] K. Schallmoser and D. Strunk, "Preparation of pooled human platelet lysate (pHPL) as an efficient supplement for animal serum-free human stem cell cultures," The Journal of Visualized Experiments, no. 32, article e1523, 2009.

[8] A. Schäffler and C. Büchler, "Concise review: adipose tissuederived stromal cells-basic and clinical implications for novel cell-based therapies," Stem Cells, vol. 25, no. 4, pp. 818-827, 2007.
[9] P. Chomczynski and N. Sacchi, "Single-step method of RNA isolation by acid guanidinium thiocyanate-phenol-chloroform extraction," Analytical Biochemistry, vol. 162, no. 1, pp. 156-159, 1987.

[10] M. W. Pfaffl, G. W. Horgan, and L. Dempfle, "Relative expression software tool (REST) for group-wise comparison and statistical analysis of relative expression results in real-time PCR," Nucleic acids research, vol. 30, no. 9, article e36, 2002.

[11] A. B. Lyons, "Analysing cell division in vivo and in vitro using flow cytometric measurement of CFSE dye dilution," Journal of Immunological Methods, vol. 243, no. 1-2, pp. 147-154, 2000.

[12] S. Baglioni, G. Cantini, G. Poli et al. et al., "Functional differences in visceral and subcutaneous fat pads originate from differences in the adipose stem cell," PLOS ONE, vol. 7, no. 5, article e36569, 2012.

[13] J. B. Mitchell, K. McIntosh, S. Zvonic et al., "Immunophenotype of human adipose-derived cells: temporal changes in stromalassociated and stem cell-associated markers," Stem Cells, vol. 24, no. 2, pp. 376-385, 2006.

[14] Y. Jiang, B. N. Jahagirdar, R. L. Reinhardt et al., "Pluripotency of mesenchymal stem cells derived from adult marrow," Nature, vol. 418, pp. 41-49, 2002.

[15] P. A. Zuk, M. Zhu, P. Ashjian et al. et al., "Human adipose tissue is a source of multipotent stem cells," Molecular Biology of the Cell, vol. 13, no. 12, pp. 4279-4295, 2002.

[16] K. Birsoy, Z. Chen, and J. Friedman, "Transcriptional regulation of adipogenesis by KLF4," Cell Metabolism, vol. 7, no. 4, pp. 339$347,2008$.

[17] M. Toyoda, Y. Matsubara, K. Lin, K. Sugimachi, and M. Furue, "Characterization and comparison of adipose tissue-derived cells from human subcutaneous and omental adipose tissues," Cell Biochemistry and Function, vol. 27, no. 7, pp. 440-447, 2009.

[18] J. M. Ryan, F. P. Barry, J. M. Murphy, and B. P. Mahon, "Mesenchymal stem cells avoid allogeneic rejection," Journal of Inflammation, vol. 2, article 8, 2005.

[19] S. Glennie, I. Soeiro, P. J. Dyson, E. W. F. Lam, and F. Dazzi, "Bone marrow mesenchymal stem cells induce division arrest anergy of activated T cells," Blood, vol. 105, no. 7, pp. 2821-2827, 2005.

[20] T. J. Burdon, A. Paul, N. Noiseux, S. Prakash, and D. ShumTim, "Bone marrow stem cell derived paracrine factors for regenerative medicine: current perspectives and therapeutic potential," Bone Marrow Research, vol. 2011, Article ID 207326, 14 pages, 2011.

[21] P. C. Baer and H. Geiger, "Adipose-derived mesenchymal stromal/stem cells: tissue localization, characterization, and heterogeneity," Stem Cells International, vol. 2012, Article ID 812693, 11 pages, 2012.

[22] M. Corselli, C.-W. Chen, M. Crisan, L. Lazzari, and B. Péault, "Perivascular ancestors of adult multipotent stem cells," Arteriosclerosis, Thrombosis, and Vascular Biology, vol. 30, pp. 11041109, 2010.

[23] R. De Siena, L. Balducci, A. Blasi et al., "Omentum-derived stromal cells improve myocardial regeneration in pig post-infarcted heart through a potent paracrine mechanism," Experimental Cell Research, vol. 316, no. 11, pp. 1804-1815, 2010.

[24] A. H. Klopp, Y. Zhang, T. Solley et al., "Omental adipose tissuederived stromal cells promote vascularization and growth of endometrial tumors," Clinical Cancer Research, vol. 18, pp. 771782, 2012. 
[25] M. Rostovskaya and K. Anastassiadis, "Differential expression of surface markers in mouse bone marrow mesenchymal stromal cell subpopulations with distinct lineage commitment," PLoS ONE, vol. 7, no. 12, article e51221, 2012. 


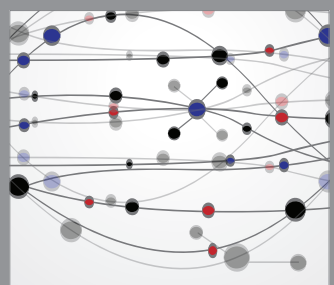

The Scientific World Journal
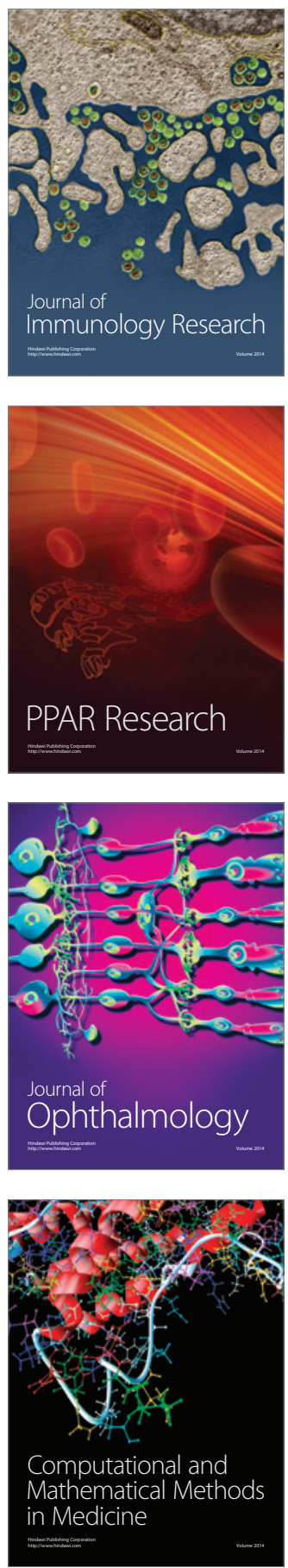

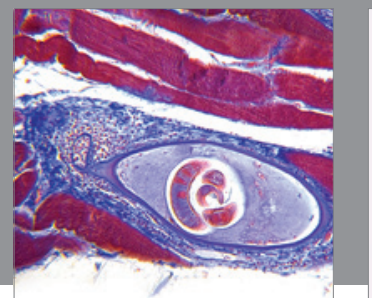

Gastroenterology

Research and Practice
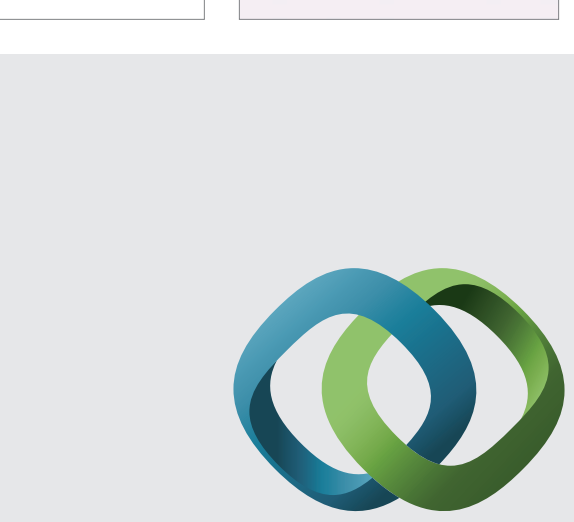

\section{Hindawi}

Submit your manuscripts at

http://www.hindawi.com
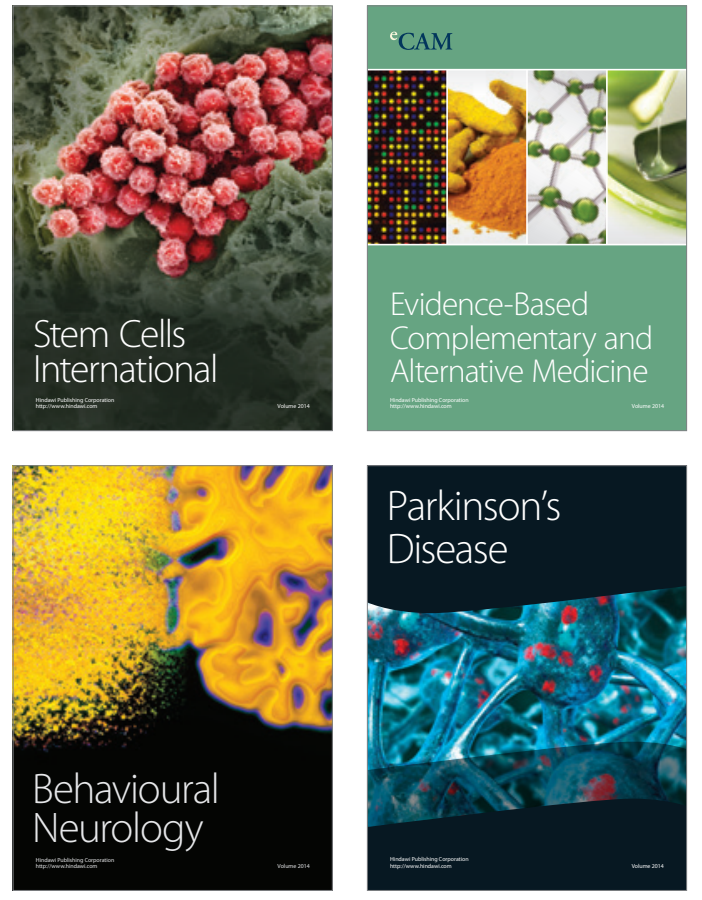
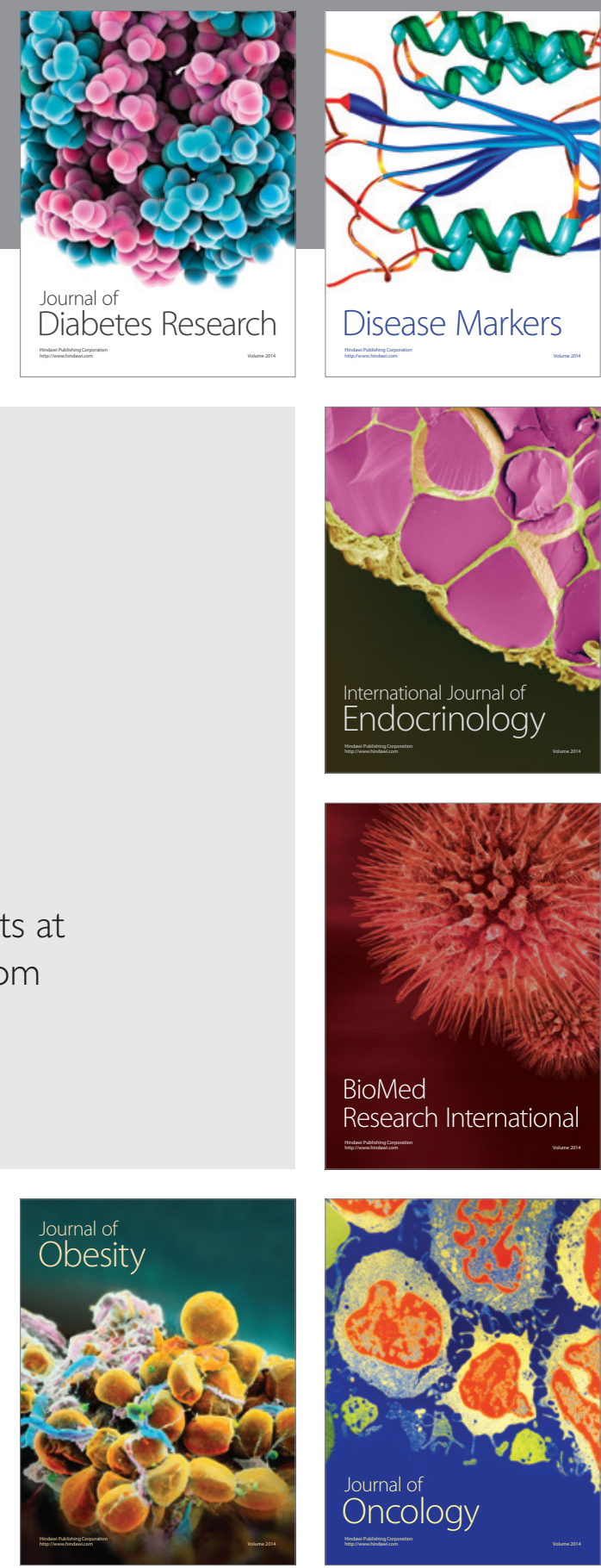

Disease Markers
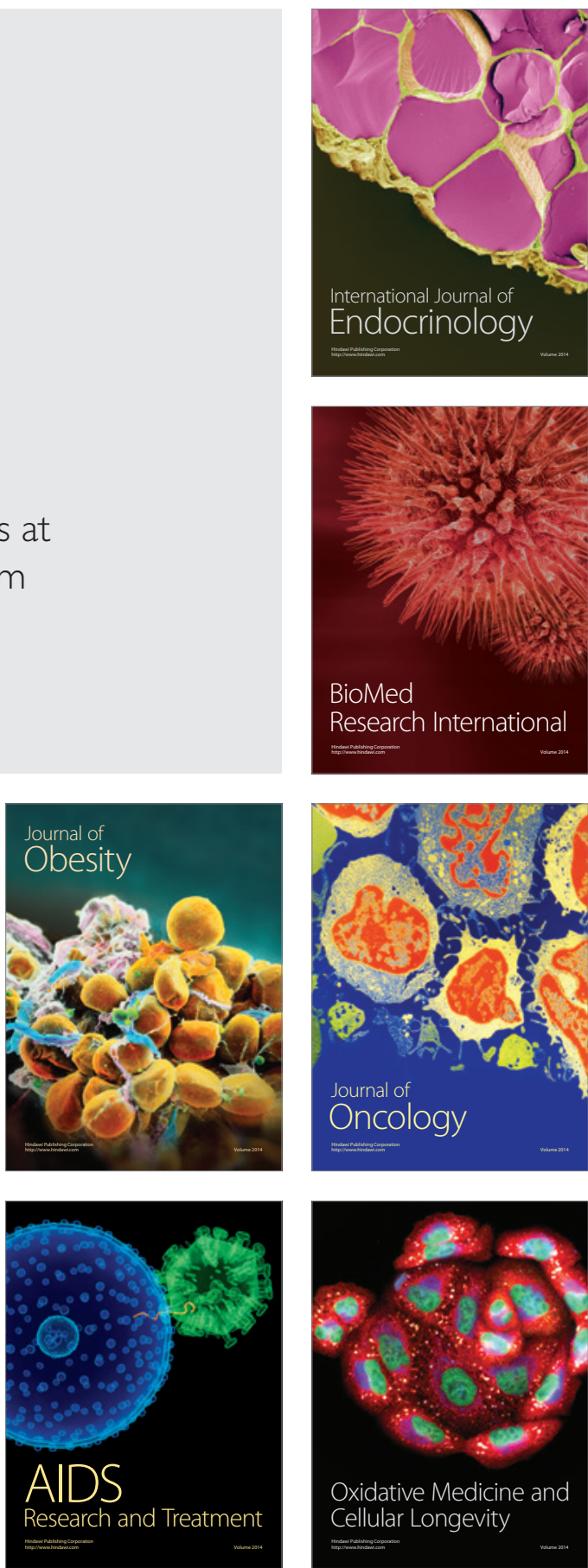